\title{
¿CÓMO POTENCIAR DESDE LA ADMINISTRACIÓN LA TRANSFERENCIA DE CONOCIMIENTO AL SECTOR AGROALIMENTARIO? EL MODELO DE INTEGRACIÓN Y COLABORACIÓN DEL PATT EN CATALUÑA
}

\author{
Martin Alba*, Lourdes Reig, David Fernández \\ CREDA UPC-IRTA (Castelldefels, martin.federico@upc.edu)
}

Palabras clave: Transferencia de conocimientos, Innovación, Políticas Públicas, Cataluña, Sector agroalimentario.

\begin{abstract}
Resumen
Este trabajo tiene por objetivo presentar los resultados del análisis de 15 años de actividad del Plan Anual de Transferencia Tecnológica (PATT) del Departamento de Acción Climática, Alimentación y Agenda Rural de la Generalitat de Cataluña. Para ello se ha realizado un estudio que integra una primera parte introductoria sobre la evolución de las actividades de este plan en cuanto al número de jornadas realizadas, número de entidades promotoras colaboradoras y el número de asistentes a dichas jornadas. Y una segunda parte con un trabajo de campo, que integró entrevistas semiestructuradas a actores clave de este modelo y una encuesta respondida por más de 700 usuarios. Los resultados encontrados han sido muy positivos en cuanto al funcionamiento del programa, a su valoración y a los posibles impactos que genera. También ha aportado algunos elementos importantes de mejora, principalmente de capitalización del conocimiento en innovación. Este trabajo presenta elementos muy útiles para los actores que conforman los sistemas de innovación agroalimentarios para potenciar la difusión de conocimiento y muestra elementos clave para que las administraciones diseñen e implementen modelos eficaces y colaborativos con ese fin ${ }^{33}$.
\end{abstract}

\section{Introducción y objetivos}

El objetivo de este trabajo ha sido realizar una evaluación global del Plan Anual de Transferencia Tecnológica (PATT) del Departamento de Acción Climática, Alimentación y Agenda Rural (DACC) de la Generalitat de Catalunya desde la visión de los principales actores involucrados como así también de los usuarios/beneficiarios de este programa.

El objetivo general del PATT consiste en promover la divulgación y transferencia de conocimientos técnicos y de gestión relacionados con el ámbito agroalimentario catalán con el fin de mejorar e incrementar la profesionalización, la innovación y la competitividad del sector agroalimentario, forestal y del medio rural de Cataluña.

Hay un amplio consenso en el sistema de innovación agroalimentario de Cataluña que actualmente existe un gap importante entre el conocimiento generado en el subsistema de conocimiento y el que finalmente es difundido/transferido al sector productivo. Es en este gap donde el PATT focaliza sus esfuerzos para reducirlo. Los objetivos del PATT pueden clasificar en:

- De corto plazo: mejorar la transferencia de información y de conocimientos entre los actores del sistema de innovación agroalimentario.

- De medio plazo: mejorar la profesionalización de los actores del sector en Cataluña.

- De largo plazo: incrementar la competitividad y la innovación del sector en Cataluña.

El DACC elabora anualmente este plan, el cual incluye actuaciones demostrativas y actividad puntuales de divulgación y transferencia de conocimientos técnicos y de gestión. Para la ejecución de todas estas actividades, el DACC cuenta con la colaboración de más de 100 entidades promotoras (empresas públicas, empresas privadas, consorcios, cooperativas, agrupaciones de productores, fundaciones, grupos LEADER, otras administraciones, etc.) vinculadas al sector agrario, ganadero, agroindustrial, forestal y del medio rural y pesquero de Cataluña.

Las jornadas PATT son una vía de divulgación y transferencia de conocimientos técnicos y de gestión que incrementan la profesionalización del sector y fortalecen la competitividad e innovación del sector, dando una respuesta adecuada a las demandas, prioridades y necesidades del sector (dado que estas jornadas se organizan con un modelo bottom up). Además, fortalecen los vínculos y las interacciones entre los actores del sistema. Durante el año 2019 se realizaron en el marco del PATT 740 jornadas técnicas, con 2.670 ponencias y 29.142 asistencias (DACC, 2020).

\section{Metodología}

\footnotetext{
${ }^{33}$ Este estudio ha sido realizado gracias al apoyo del Departamento de Acción Climática, Alimentación y Agenda Rural de la Generalitat de Catalunya (DACC). Proyecto de evaluación del PATT (2017-2018).
} 
Para realizar la evaluación de este programa se ha utilizado la metodología DAC $^{34}$ desarrollada por la $\mathrm{OECD}^{35}$, que evalúa programas y políticas públicas en base a 6 criterios: Relevancia, Coherencia, Eficacia, sostenibilidad, eficiencia e Impacto. En este estudio hemos focalizado en 3 criterios: relevancia, eficacia e impacto.

Las fuentes de información han sido principalmente primarias, 17 entrevistas semiestructuras a las principales entidades promotoras de jornadas PATT (más representativas por cantidad de jornadas/asistentes: $51 \%$ de les jornadas realizadas y un 52\% del asistentes) y una encuesta respondida por 757 usuarios beneficiarios de estas jornadas (participantes en jornadas realizadas durante 2017-2018).

El perfil de los beneficiarios que contestaron la encuesta ha sido de:

- Profesionales activos del sector agroalimentario, forestal i del medio rural ( $80 \%$ de los asistentes).

- Principalmente de los ámbitos vinculados a la agricultura i la ganadería (52\%).

- De mediana edad (62\% entre 41 y 65 años).

- Principalmente de género masculino (56\% frente a un $43 \%$ femenino).

\section{Resultados}

En el cuadro 1 encontramos un resumen de los resultados principales en cuanto a relevancia, eficacia e impacto que se detallan a continuación.

\section{1) Relevancia}

La adecuación de les jornadas a les prioridades y necesidades del sector fue muy bien valorada por un $76 \%$ de las entidades promotoras (8,4 de valoración media en una escala de 1 a 10). Además, un $83 \%$ de las entidades promotoras considera que la contribución del PATT es significativa o muy significativa para promover la transferencia de conocimiento (valoración media de 7,6) y un 59\% de las personas entrevistadas opinan que las actividades del PATT son relevantes o muy relevantes para sus propias entidades promotoras (valoración media de 7,8). Sin embargo, más allá de la buena valoración comentada, algunas entidades proponen incentivar un proceso más profundo de detección de las necesidades del sector y potenciar los instrumentos de asesoramiento, extensión y transferencia tecnológica. En otras palabras, las jornadas se consideran un instrumento adecuado pero complementario a otros instrumentos de transferencia tecnológica.

En cuanto a los usuarios beneficiarios, el PATT tiene una muy buena valoración: 93\% de los asistentes a las jornadas está satisfecho o muy satisfecho con las jornadas (valoración media de 8,2). Además, un 74\% de los asistentes participa en dos o más jornadas anualmente (21\% en más de 4 jornadas y $53 \%$ entre dos y tres jornadas). Por otra parte el $90 \%$ de las personas encuestadas considera útiles o muy útiles las jornadas PATT como fuente de nuevos conocimientos e información (valoración media de 7,8).

\section{2) Eficacia}

La eficacia del PATT como instrumentos de transferencia también ha obtenido una valoración muy positiva, el 77\% de las entidades promotoras valoran positivamente su eficacia (valoración media de 7,8). Sin embargo, se considera que el PATT es un instrumento eficaz de difusión de conocimiento y divulgación, pero no de transferencia tecnológica propiamente dicha (con implementación real por parte del sector).

A nivel de usuarios, un $87 \%$ de los asistentes valora positiva o muy positivamente las jornadas como fuente principal de conocimiento (valoración media de 7,8). Por otro lado, un $72 \%$ de las personas encuestadas afirma que aplican los conocimientos adquiridos en las jornadas con bastante o mucha frecuencia $(7,8$ de valoración media).

\section{3) Impacto}

La percepción de efectos e impactos positivos de las jornadas por parte de las entidades promotoras también ha obtenido valoraciones muy positivas: un $80 \%$ de las entidades considera que las jornadas han tenido un impacto alto o muy alto sobre las propias entidades, principalmente por el incremento de relaciones con otros profesionales y por los conocimientos relevantes transferidos y las capacidades generadas.

\footnotetext{
${ }^{34}$ Development Assistance Committee (DAC)

${ }^{35} \mathrm{https}$ ://www.oecd.org/dac/evaluation/daccriteriaforevaluatingdevelopmentassistance.htm
} 
Cuadro 5. Resumen de los resultados de la evaluación del PATT

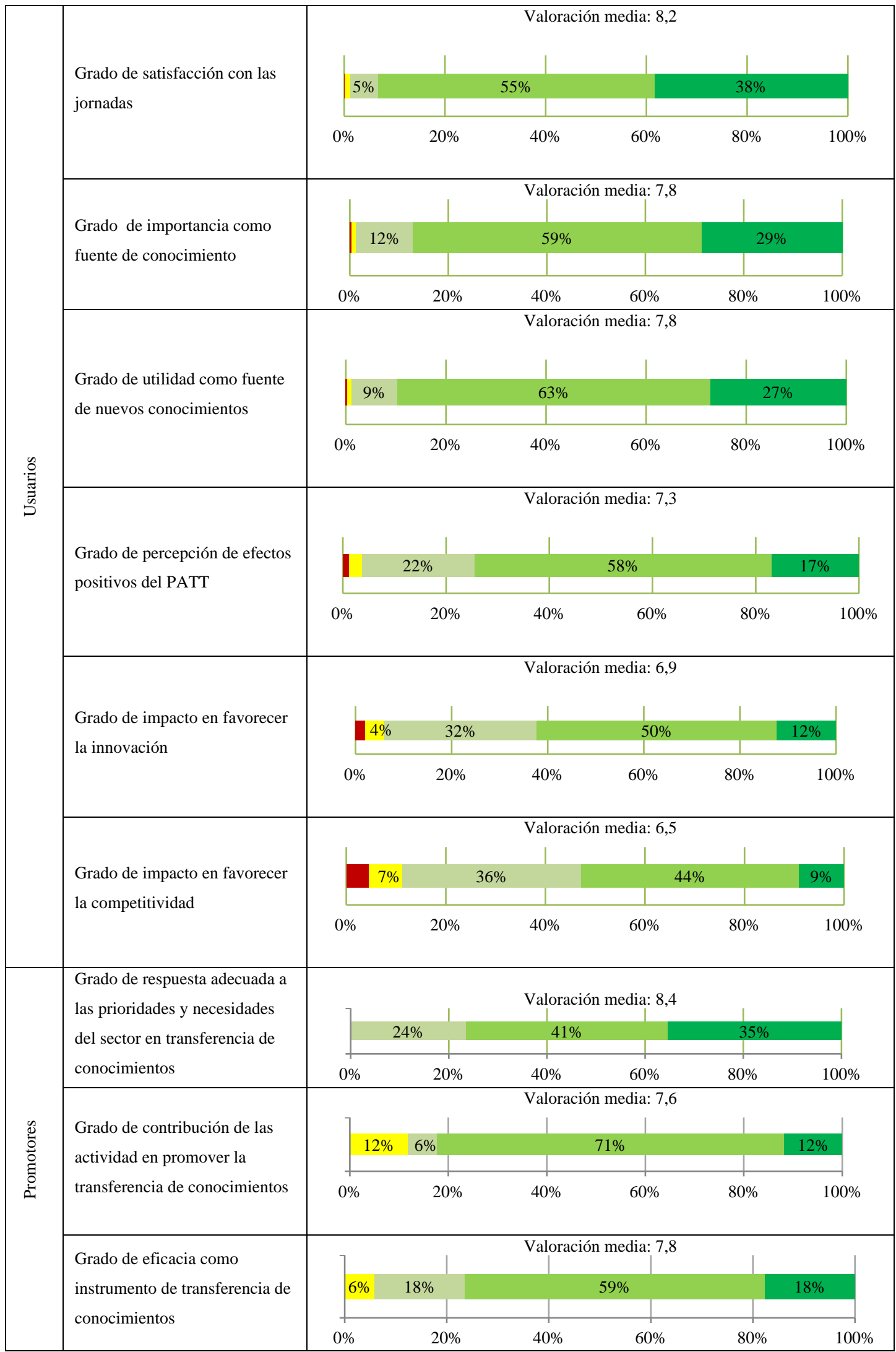

Fuente: Elaboración propia

A nivel de usuarios, un 70\% de los asistentes considera que las jornadas mejoraron su nivel de actualización (nuevos temas tecnológicos, normativos y de mercados) y su propio desarrollo profesional. Además, un 
$75 \%$ de los asistentes consideró que las jornadas PATT, han tenido repercusiones positivas en sus respectivas organizaciones (valoración media de 7,3). Por otro lado, un 62\% de los asistentes consideró que las jornadas PATT favorecen la innovación en sus respectivas organizaciones (valoración media de 6,9). Finalmente un 53\% de los asistentes ha considerado que el PATT mejora la competitividad de sus empresas (valoración media de 6,5). La adaptación a los cambios normativos $(6,8)$, la mejora de las colaboraciones con otras entidades $(6,4)$ y la mejora de los métodos organizativos y productivos $(6,4)$ son las actividades $\mathrm{y}$ aspectos mejor puntuados a nivel de impactos percibidos.

\section{Conclusiones}

Los resultados del proceso de evaluación del PATT muestran la importancia de este programa como instrumento de difusión de conocimientos en el sector agroalimentario de Cataluña, tanto por las entidades promotoras como por los usuarios asistentes a estas jornadas. La evaluación realizada muestra resultados muy positivos en los siguientes criterios utilizados:

- Relevancia: un $83 \%$ de las entidades promotoras considera que la contribución del PATT es significativa o muy significativa para promover la trasferencia de conocimiento y un $93 \%$ de los usuarios se declara satisfecho o muy satisfecho con las jornadas.

- Eficacia: las jornadas PATT son la fuente de conocimiento más valorada por los asistentes y un $71 \%$ aplican los conocimientos adquiridos.

- Impacto: un 75\% de los asistentes considera que el PATT ha tenido efectos positivos sobre sus entidades, un $62 \%$ consideró que ha favorecido la innovación y un 53\% que han mejorado la competitividad de las empresas que han participado.

- Además, se ha remarcado la importancia que tienen estas jornadas como punto de encuentro que potencia la visualización de las diversas entidades y la socialización dentro del sector, muy útil para fortalecer los proyectos en ejecución.

Sin embargo, hay puntos mejorables, el impacto aunque se considera positivo se visualiza como insuficiente por varios factores limitantes entre los que se destacan: acceso a información mejorable, alcance sectorial limitado y finalmente el impacto depende en gran medida del propio perfil del asistente/usuario. Además, es importante destacar que las jornadas son un instrumento complementario de la transferencia tecnológica, pero no puede ser el único, considerando muy importante reforzar otros instrumentos como los de asesoramiento, extensión y transferencia.

\section{Bibliografía}

- DACC (2020). Memoria PRITAC 2019. DACC.

- Enzing, C.; Percy-Smith, A.; Dani, S.; Hinojosa, C.; Ploeg, M. (2015). CTA's Science, Technology and Innovation Programme (2010-2014). CTA - Technopolis.

- $\quad$ OECD (2021). Applying Evaluation Criteria Thoughtfully. OECD Publishing, Paris.

\section{Agradecimientos}

Los autores agradecen el apoyo recibido por el Departamento de Acción Climática, Alimentación y Agenda Rural de Cataluña en este proyecto y por la colaboración de todo el equipo de trabajo en especial: María Aurell y Anna Sagrera del equipo del CREDA UPC-IRTA y Maria Josep de Ribot y Victor Miguel Perez del equipo del Servicio de Innovación Agroalimentaria del DACC. 